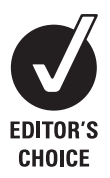

${ }^{1}$ Centre for Health Economics, University of York, York, UK;

${ }^{2}$ Centre for Reviews and Dissemination, University of York, York, UK; ${ }^{3}$ Yorkshire Heart Centre, Leeds, UK; ${ }^{4}$ The Cardiothoracic Centre, Liverpool NHS Trust, UK

Correspondence to: Dr Claire McKenna, Centre for Health Economics, Alcuin 'A' Block, University of York, Heslington, York Y010 5DD, UK: cm535@york.ac.uk

Accepted 24 November 2008 Published Online First 18 December 2008

\title{
Cost-effectiveness of radiofrequency catheter ablation for the treatment of atrial fibrillation in the United Kingdom
}

\author{
C McKenna, ${ }^{1}$ S Palmer, ${ }^{1}$ M Rodgers, ${ }^{2}$ D Chambers, ${ }^{2}$ N Hawkins, ${ }^{1}$ S Golder, ${ }^{2}$ S Van \\ Hout, ${ }^{1}$ C Pepper, ${ }^{3}$ D Todd, ${ }^{4}$ N Woolacott ${ }^{2}$
}

\begin{abstract}
Objective: To assess the cost-effectiveness of radiofrequency catheter ablation (RFCA) compared with antiarrhythmic drug (AAD) therapy for the treatment of atrial fibrillation (AF) from the perspective of the UK NHS.
\end{abstract}

Design: Bayesian evidence synthesis and decision analytical model.

Methods: A systematic review and meta-analysis was conducted and Bayesian statistical methods used to synthesise the effectiveness evidence from randomised control trials. A decision analytical model was developed to assess the costs and consequences associated with the primary outcome of the trials over a lifetime time horizon.

Main outcome measure: Costs from a health service perspective and outcomes measured as quality-adjusted life years (QALYS).

Results: The incremental cost-effectiveness ratio of RFCA varied between $£ 7763$ and $£ 7910$ for each additional QALY according to baseline risk of stroke, with a probability of being cost-effective from 0.98 to 0.99 for a cost-effectiveness threshold of $£ 20000$. Results were sensitive to the duration of quality of life benefits from treatment.

Conclusions: RFCA is potentially cost-effective for the treatment of paroxysmal AF in patients' predominantly refractory to $A A D$ therapy provided the quality-of-life benefits from treatment are maintained for more than 5 years. These findings remain subject to limitations in the existing evidence regarding the nature of life benefits and the prognostic importance of restoring normal sinus rhythm conferred using RFCA.

Atrial fibrillation (AF) is a common and debilitating cardiac arrhythmia. Its prevalence in the United Kingdom is rising as a result of an ageing population and an increased longevity resulting from improved medical care for chronic cardiac conditions which predispose to $\mathrm{AF} .{ }^{1} \mathrm{~A}$ recent report estimated that approximately $1 \%$ of all NHS expenditures are the result of $\mathrm{AF}^{2}$ The costeffective management of AF in the United Kingdom therefore represents an important consideration

Radiofrequency catheter ablation (RFCA) is an established technique for the treatment of cardiac arrhythmias, which has relatively recently been extended to allow treatment of AF. In the United Kingdom, its use for controlling AF is recommended by the National Institute for Health and Clinical Excellence (NICE) for patients who have previously failed on other management strategies. ${ }^{3}$
RFCA offers the potential to eliminate the underlying cause of AF, which other therapies fail to achieve. The primary outcome from the procedure is the restoration and maintenance of normal sinus rhythm (NSR). The sustained long-term maintenance of sinus rhythm establishes the efficacy of the intervention.

RFCA results in significant upfront costs and a risk of procedural complications. However, the potential quality-of-life benefits through long-term absence of arrhythmia and any associated prognostic benefits (for example, reduced risk of stroke) may outweigh the costs when compared to longterm use of anti-arrhythmic drug (AAD) therapy and its associated adverse effects. Only one previous study, based on a US population, has examined the cost-effectiveness of RFCA for AF. ${ }^{4}$ Our aim was to develop a UK-specific costeffectiveness model of RFCA compared with $\mathrm{AADs}$ for patients predominantly refractory to at least one previous $\mathrm{AAD}$.

\section{METHODS}

\section{Overview}

A probabilistic decision analytical model was developed to assess the cost-effectiveness of RFCA in the UK National Health Service. A systematic review and meta-analysis was conducted and Bayesian statistical methods used to synthesise the effectiveness evidence from randomised control trials (RCTs). The model considers the short-term and long-term costs and consequences associated with the primary outcome of the trials: freedom from arrhythmia at 12 months.

The structure of the decision analytical model and its underlying assumptions were developed in discussion with UK clinical advisers. Outcomes in the model were expressed in terms of qualityadjusted life years (OALYs). Costs were considered from the perspective of the UK NHS and Personal Social Services, using 2006 prices, with costs and benefits discounted at $3.5 \%$ per year. ${ }^{5}$

\section{Treatment strategies and population}

The decision model evaluates the cost-effectiveness of RFCA in adults with AF refractory to at least one $\mathrm{AAD}$. It evaluates a strategy of RFCA (without long-term $\mathrm{AAD}$ use) compared to long-term $\mathrm{AAD}$ treatment alone. The AAD considered is amiodarone, selected on the basis that this drug is most likely to be given after patients have previously failed on other AADs in routine practice. The 
A

Stroke

M

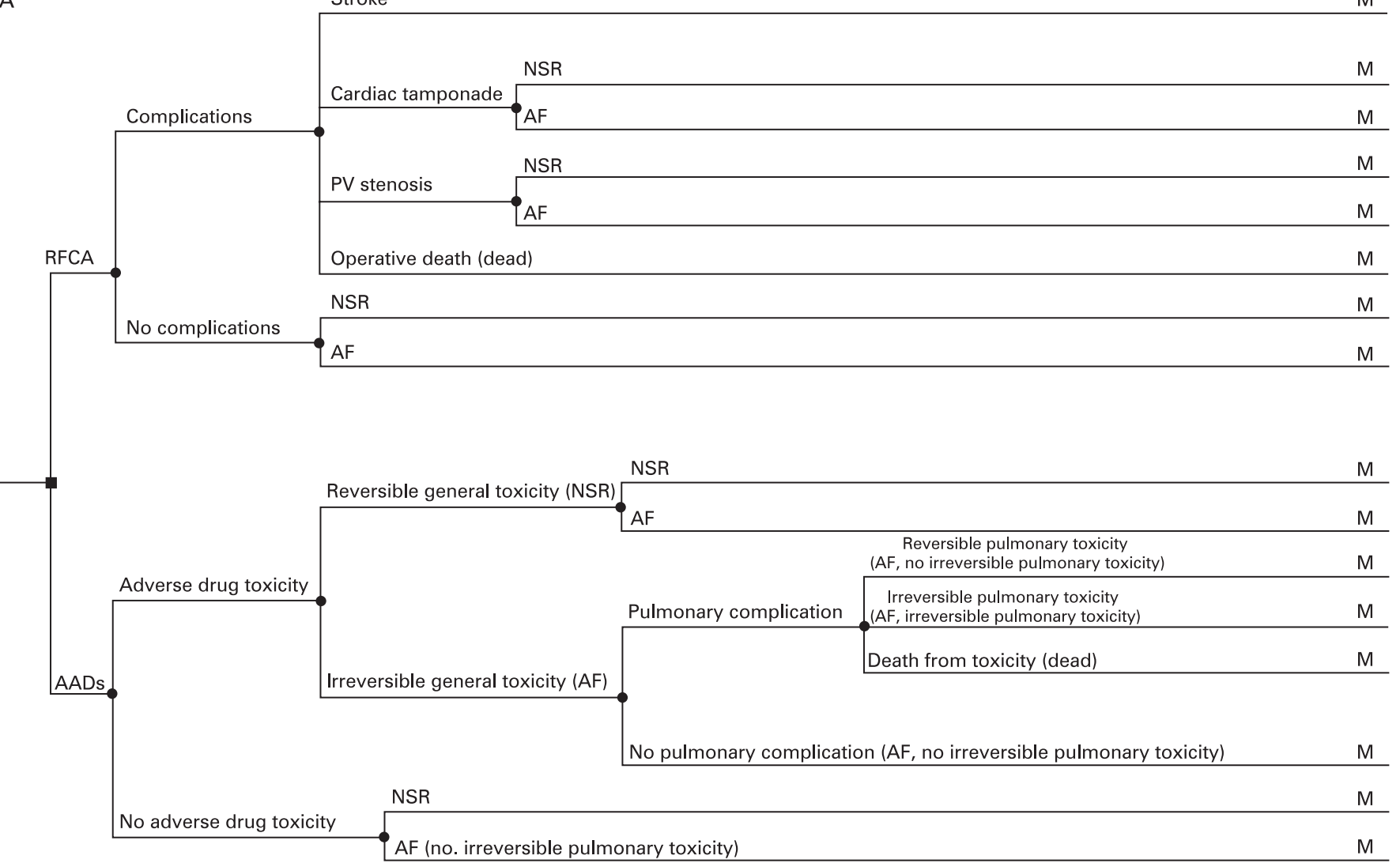

B

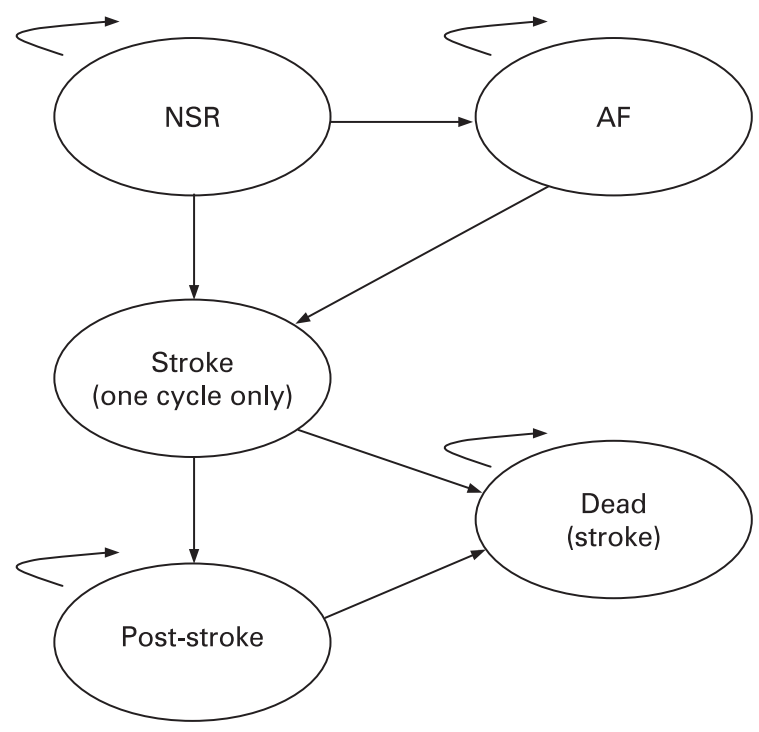

Figure 1 (A) Structure of the short-term model. Schematic of pathways leading to the two main starting health states (normal sinus rhythm (NSR)/ atrial fibrillation (AF)) of the long-term Markov model. (B) Structure of the long-term Markov model. Additional states were incorporated for the antiarrhythmic drug (AAD) strategy because of potential adverse toxicity events.

majority of subjects included in the RCTs had paroxysmal AF as opposed to persistent or permanent forms.

\section{Model structure}

The decision model comprised two components: a decision tree that captured the short-term clinical outcomes and costs associated with the treatment strategies up to a period of 12 months; and a long-term Markov model, which extrapolates the costs and outcomes over a lifetime time horizon using annual cycles. Figure 1 provides a schematic of the model structure.

For RFCA, the risk of operative death or procedural complications, including cardiac tamponade, stroke and pulmonary vein (PV) stenosis, were modelled. For AADs, the risk of adverse drug toxicity (both reversible and irreversible) was considered. In addition, a risk of pulmonary complications given withdrawal from treatment because of an acute episode of toxicity, was modelled. An elevated risk of mortality was also 
assumed to be associated with an irreversible event. Several competing risks including stroke, adverse bleeding events (due to concomitant medications), and other causes of mortality were also included in the short-term model for both treatment strategies. At the end of the 12-month period, the short-term model established the proportion of patients that entered the two main starting health states of the long-term extrapolation model, after accounting for mortality and the risk of stroke: NSR (that is, restoration to normal sinus rhythm) and AF (that is, reversion to atrial fibrillation).

The long-term model took the form of a Markov process with health states for NSR, AF, stroke and death. The potential adverse drug toxicity events associated with the use of AADs required additional states in the $\mathrm{AAD}$ model. Patients in the NSR state faced an annual probability of reversion back to AF and an elevated risk of stroke compared to the general population. Patients in the AF state were assumed to be withdrawn from treatment and faced a higher risk of stroke than NSR patients. Patients surviving the first year of a stroke entered a post-stroke state, where the risk of death due to stroke and the costs incurred from stroke were lower than in the first year of the event. In each yearly cycle, all patients regardless of their current health state faced an annual risk of mortality from other causes (non-stroke mortality). In addition, the model included a cost and quality-of-life decrement associated with the risk of major and minor bleeding events from the use of concomitant medications.

\section{Model inputs}

\section{Clinical effectiveness}

Three separate analyses considering a range of alternative data sources were used to inform the clinical effectiveness inputs. The primary health outcome considered was freedom from AF at 12 months. These data were used to estimate the probability of NSR and AF for RFCA and AADs applied in the short-term model. Three randomised controlled trials (RCTs) were identified where RFCA was compared directly to AADs for patients with predominantly paroxysmal $A F .{ }^{6-8}$ In the absence of a single study with which to populate a UK baseline event rate for RFCA, evidence from a wider range of case series and survey data were considered. A number of individual case series were identified that reported the primary outcome at 12 months following RFCA. In addition, a worldwide survey was identified on the efficacy of RFCA. ${ }^{9}$

Bayesian meta-analytical approaches were used to synthesise the RCT evidence and the non-RCT data. A random baseline, fixed-effect model was used as the basis for the meta-analysis. Outcomes were estimated as freedom from AF by combining the weight of evidence from the RCTs and the external evidence. The base-case analysis establishes the cost-effectiveness of RFCA on the assumption that the average patient is treated as in the RCTs. Alternative scenarios were undertaken by combining the RCT evidence with the case series and survey data.

Table 1 summarises the main input variables and sources applied in the cost-effectiveness model. The various components are described below.

\section{Long-term reversion rates (NSR to AF)}

Central to the long-term model was the subsequent event rates (and costs and quality-of-life estimates) for patients who left the short-term model free of arrhythmia (NSR) or not (AF) at 12 months. The long-term reversion rates to AF represent important parameters because any additional benefit assigned to NSR relative to AF is only maintained in the long term if patients continue to remain free of arrhythmia. In the absence of data from the RCTs of RFCA beyond 12 months of followup, these estimates were obtained from other sources. The annual rate of revision for patients who receive RFCA was estimated from a large controlled study with a median followup of 900 days. ${ }^{10}$ The annual reversion rate for patients receiving AADs was estimated from a multicentre trial examining the long-term efficacy of amiodarone in preventing recurrent AF. ${ }^{11}$

\section{Stroke}

The baseline risk of stroke for AF was based on the $\mathrm{CHADS}_{2}$ index, which has been validated in a national registry of $\mathrm{AF} .{ }^{12} \mathrm{~A}$ numerical score is given to each of five risk factors (recent congestive heart failure, hypertension, age, diabetes mellitus, history of stroke or transient ischaemic attack), and the total score $(\leqslant 6)$ equates to a stroke risk for $\mathrm{AF}$. $\mathrm{CHADS}_{2}$ is broadly similar to the risk stratification algorithm proposed by the National Institute for Health and Clinical Excellence (NICE), which stratifies subjects into low, moderate and high-risk categories. ${ }^{13}$

Consideration was also given as to whether the stroke risk was different according to NSR/AF status. No direct evidence from the RCTs was available to quantify the potential differential risk for NSR/AF. A separate search of the literature was undertaken to identify additional evidence related to the prognostic value of NSR in patients with AF. The search identified an analysis of the AFFIRM study, which examined the occurrence and characteristics of stroke events in their investigation of sinus rhythm management, and provided an estimate of the hazard of stroke for AF relative to NSR. ${ }^{14}$ Using a Cox proportional hazards regression analysis, the presence of AF was found to be significantly associated with a $60 \%$ increase in the risk of stroke after adjusting for several covariates including the use of warfarin therapy. This provided an estimate of the stroke risk reduction for NSR, which was lower than the risk for AF but remained higher than the general population.

To reduce thromboembolism in AF, most patients, regardless of treatment strategy, receive some form of anticoagulants or antiplatelets. The Euro Heart Survey on AF analysed current antithrombotic drug prescriptions. ${ }^{15}$ These data were used to estimate the proportion of patients likely to receive warfarin, aspirin or no oral anticoagulants (OACs) in the United Kingdom. Stroke risk reduction through the use of OACs was derived from a systematic review and meta-analysis of stroke prevention with warfarin and aspirin in patients with AF. ${ }^{13}$ The adjustments were applied to the stroke risk for NSR and AF derived from the $\mathrm{CHADS}_{2}$ index.

\section{Mortality}

The model separates deaths into those caused by stroke, drug toxicity and other cause mortality. The mortality risk from stroke was derived from a UK-based Community Stroke Project, which examined the long-term prognosis after acute stroke. ${ }^{16}$ The age-dependent risk of other-cause mortality was based on standard UK age-specific and sex-specific mortality rates. ${ }^{17}$ The treatments were assumed not to infer a differential mortality effect, except through their reduction in the risk of stroke through NSR or AF.

\section{Resource use and unit costs}

The resources considered were based on the short-term and long-term events associated with each treatment strategy. The 
Table 1 Summary of input parameters applied in the base-case model

\begin{tabular}{|c|c|c|c|c|c|}
\hline Variables & Value & Low CI & High Cl & Distribution & Ref \\
\hline \multicolumn{6}{|l|}{ Probability of NSR at 1 year } \\
\hline RFCA & 0.8405 & 0.5579 & 0.9631 & Posterior & \\
\hline AADs & 0.3682 & 0.1060 & 0.7083 & Posterior & \\
\hline Odds ratio & 0.0968 & 0.0520 & 0.1641 & Posterior & \\
\hline \multicolumn{6}{|l|}{ Annual probability of recurrent AF after year 1} \\
\hline RFCA & 0.0335 & 0.0220 & 0.0451 & Beta & 10 \\
\hline AADs & 0.2883 & 0.2323 & 0.3456 & Beta & 11 \\
\hline \multicolumn{6}{|l|}{ Stroke risk for AF (\%) } \\
\hline $\mathrm{CHADS}_{2}=0$ & 1.9 & 1.2 & 3.0 & Beta & 12 \\
\hline $\mathrm{CHADS}_{2}=1$ & 2.8 & 2.0 & 3.8 & Beta & 12 \\
\hline $\mathrm{CHADS}_{2}=2$ & 4.0 & 3.1 & 5.1 & Beta & 12 \\
\hline $\mathrm{CHADS}_{2}=3$ & 5.9 & 4.6 & 7.3 & Beta & 12 \\
\hline Hazard ratio relative to $\mathrm{NSR}^{*}$ & 1.60 & 1.11 & 2.30 & Log normal & 14 \\
\hline \multicolumn{6}{|l|}{ Stroke risk reduction with OACs (RR) } \\
\hline Warfarin relative to placebo & 0.33 & 0.24 & 0.45 & Log normal & 13 \\
\hline Warfarin relative to aspirin & 0.59 & 0.40 & 0.86 & Log normal & 13 \\
\hline \multicolumn{6}{|l|}{ OAC use (\%) } \\
\hline Warfarin & 64.0 & - & - & Dirichlet & 15 \\
\hline Aspirin & 27.3 & - & - & Dirichlet & 15 \\
\hline None & 8.7 & - & - & Dirichlet & 15 \\
\hline \multicolumn{6}{|l|}{ Mortality risk from stroke (RR) } \\
\hline In year 1 & 7.40 & 6.50 & 8.50 & Betapert & 16 \\
\hline In subsequent years & 2.30 & 2.00 & 2.70 & Betapert & 16 \\
\hline \multicolumn{6}{|l|}{ Adverse toxicity from AADs (\%) } \\
\hline General toxicity (year 1) & 12.50 & 10.00 & 15.00 & Betapert & 22 \\
\hline General toxicity ( $\geqslant 2$ years) & 6.25 & 5.00 & 7.50 & Betapert & 22 \\
\hline Withdrawal due to toxicity (year 1 ) & 10.00 & 6.25 & 12.50 & Betapert & 22 \\
\hline Withdrawal due to toxicity ( $\geqslant 2$ years) & 5.00 & 3.13 & 6.25 & Betapert & 22 \\
\hline Pulmonary complication given withdrawal & 15.19 & 1.00 & 30.00 & Betapert & 22 \\
\hline Irreversible pulmonary complication & 25.00 & 0.00 & 30.00 & Betapert & 22 \\
\hline Mortality from irreversible pulmonary event & 20.00 & 5.00 & 25.00 & Betapert & 22 \\
\hline \multicolumn{6}{|l|}{ Adverse bleeding from OACs (\%) } \\
\hline Major bleed on warfarin & 2.40 & 1.70 & 8.10 & Betapert & 26 \\
\hline Minor bleed on warfarin & 15.80 & 15.00 & 16.60 & Betapert & 26 \\
\hline \multicolumn{6}{|l|}{ Bleeding risk reduction on aspirin (RR) } \\
\hline Major bleed & 0.58 & 0.35 & 0.97 & Log normal & 13 \\
\hline Minor bleed & 0.45 & 0.32 & 0.64 & Log normal & 13 \\
\hline \multicolumn{6}{|l|}{ Bleeding risk reduction on no OACs (RR) } \\
\hline Major bleed & 0.45 & 0.25 & 0.82 & Log normal & 13 \\
\hline Minor bleed & 0.46 & 0.36 & 0.59 & Log normal & 13 \\
\hline \multicolumn{6}{|l|}{ RFCA procedural complications (\%) } \\
\hline Operative death & 0.05 & 0.00 & 0.09 & Beta & 9 \\
\hline Cardiac tamponade & 1.22 & 0.99 & 1.45 & Beta & 9 \\
\hline Stroke & 0.28 & 0.16 & 0.40 & Beta & 9 \\
\hline PV stenosis & 0.74 & 0.54 & 0.94 & Beta & 9 \\
\hline Mean number of procedures per patient & 1.304 & 1.293 & 1.315 & Normal & 9 \\
\hline Utilities associated with health states & & SE & & & \\
\hline Decrement for NSR given RFCA & 0.0000 & - & 30 & - & 27 \\
\hline Decrement for NSR given AADs & 0.0199 & 0.0100 & - & Gamma & 27 \\
\hline Decrement for AF given RFCA & 0.0034 & 0.0017 & - & Gamma & 28 \\
\hline Decrement for AF given AADs & 0.0925 & 0.0361 & - & Gamma & 28 \\
\hline Disabled stroke & 0.38 & 0.0460 & - & Beta & 19 \\
\hline Non-disabled stroke & 0.74 & 0.0260 & - & Beta & 19 \\
\hline Combined stoke (30.9\% disabled) & 0.63 & - & - & - & 19 \\
\hline Decrement for pulmonary toxicity & 0.0329 & 0.0030 & - & Gamma & 21 \\
\hline $\begin{array}{l}\text { Decrement for non-pulmonary toxicity or bleeding } \\
\text { event (days of perfect health lost) }\end{array}$ & 1 & 0 & - & Betapert & 22 \\
\hline \multicolumn{6}{|l|}{ Unit costs (f) } \\
\hline RFCA accumulated cost $\dagger$ & 9810 & - & - & Fixed & \\
\hline Complication from cardiac tamponade & 815 & - & - & Fixed & 18 \\
\hline Complication from PV stenosis & 3217 & - & - & Fixed & 18 \\
\hline Outpatient initiation of amiodarone & 154 & - & - & Fixed & 18 \\
\hline Amiodarone (200 mg daily, per annum) & 32 & - & - & Fixed & 29 \\
\hline
\end{tabular}


Table 1 Continued

\begin{tabular}{lllllc}
\hline Variables & Value & Low Cl & High Cl & Distribution & Ref \\
\hline AF health state (per annum) & 646 & - & - & Fixed & 2 \\
NSR health state (per annum) & 646 & - & - & Fixed & 2 \\
Stroke (year 1) & 9431 & - & - & Fixed & 19 \\
Stroke ( $\geqslant 2$ years, per annum) & 2488 & - & - & Fixed & 19 \\
Warfarin (5 mg daily, per annum) & 19 & - & - & Fixed & 29 \\
Aspirin (75 mg daily, per annum) & 20 & - & - & Fixed & 29 \\
Toxic event & 1497 & - & - & Fixed & 30 \\
Reversible toxicity (per day) & 0.43 & - & - & Fixed & 29 \\
Irreversible toxicity (50 mg daily) & 158 & - & - & Fixed & 29 \\
Major bleeding event & 1573 & - & - & Fixed & 26 \\
Minor bleeding event & 87 & - & - & - & - \\
\hline
\end{tabular}

$\mathrm{AAD}$, anti-arrhythmic drug; $\mathrm{AF}$, atrial fibrillation; $\mathrm{CHADS}_{2}$, congestive heart failure, hypertension, age 75, diabetes mellitus, and prior stroke; Cl, confidence interval or credible interval; NSR, normal sinus rhythm; OAC, oral anticoagulants; PV, pulmonary vein; RFCA, radiofrequency catheter ablation; $R R$, relative risk; SE, standard error.

*Used to determine the stroke risk for NSR. The hazard ratio is applied to the CHADS 2 stroke risk scores for AF.

$\nmid$ RFCA procedural cost consists of total consumable ( $£ 5687$ ), ward ( $\mathrm{f} 182$ based on 2 days), and lab ( $f 1979$ based on

200 minutes) costs plus VAT and administration costs (Fitzpatrick A, personal communication, 2007).

short-term costs associated with RFCA include those related to the initial procedure, repeat procedures and the management of complications. In the absence of suitable national reference cost estimates for the procedure in the UK NHS, the cost of RFCA in AF was estimated based on a detailed study of the costs of consumables, ward (2 days) and lab time (200 minutes) undertaken by a UK cardiologist (Fitzpatrick A, personal communication, 2007). The costs of procedural related complications were derived from reference cost schedules. ${ }^{18}$ The short-term costs associated with AADs comprised the drug acquisition and administration costs of amiodarone including the management of adverse effects. Amiodarone (200 mg daily) was assumed to be initiated in an outpatient setting. In addition to ongoing intervention costs and other related costs (including OACs and the management of toxic events), annual costs for the main health states in the Markov model were applied. Costs for the two underlying AF health states (NSR and AF) were estimated from a recent study examining the cost of AF in the United Kingdom. ${ }^{2}$ This study estimates the costs of community and hospital-based care related to AF, including general practitioner consultations, anticoagulation visits and hospital costs. In the absence of cost data which discriminated between the NSR and AF states, a conservative assumption towards RFCA was applied by applying the same annual costs to both states over the lifetime horizon. An annual cost associated with stroke was applied to the stroke state with a higher cost applied in the first year of the event. ${ }^{19}$

\section{Quality of life adjustment}

In order to estimate OALYs, it is necessary to quality adjust the period of time the average patient is alive within the model using an appropriate utility or preference score. In the absence of utility data from the trials, external data sources were sought to quantify the differential impact of the treatment regimens in terms of quality of life ( $\mathrm{OoL})$ and to differentiate patient health status according to the different states of the model. A comprehensive literature review was undertaken but, despite consideration of a large number of studies, no single source was identified that would provide a relevant source of baseline utility on which to apply incremental changes in utility according to health status. To encapsulate a baseline value in the model, the underlying utility of the general population, derived from a nationally representative UK sample using EQ5D, was used as a reference point..$^{20}$ Patients restored to NSR following RFCA (estimated to be associated with the largest improvement in utility) were assumed to revert to having the same QoL as the general population. For the other main health states, specific decrements were estimated (that is, AF following RFCA and NSR/AF following AADs) relative to the utility value estimated for patients restored to NSR following RFCA. These decrements were then applied to the general population utility values assumed to represent the QoL in the NSR state following RFCA. In addition, a single utility score for stroke was applied. ${ }^{19}$ Utility decrements were also applied for irreversible pulmonary toxicity, other general side effects and for major and minor bleeding events. ${ }^{21} 22$

\section{Analytical methods}

The model was run probabilistically using Monte Carlo simulation and the uncertainty in the individual parameters fully characterised using the probability distributions summarised in table 1 . The results are presented in two ways. First, mean lifetime costs and OALYs of both strategies are presented and their cost-effectiveness compared using incremental costeffectiveness ratios (ICERs). ${ }^{23}$ Second, decision uncertainty is presented as the probability that each strategy is considered the more cost-effective option for a given cost-effectiveness threshold.

The following analyses are undertaken for an average starting age of 52 years and $80 \%$ of subjects being male (typical of the RFCA population).$^{24}$ First, heterogeneity in patients is explored by undertaking separate analyses according to different baseline risks of stroke given by the $\mathrm{CHADS}_{2}$ score. In the base-case analysis, $\mathrm{CHADS}_{2}$ scores between 0 and 3 are considered. Separate analyses are also undertaken within the base-case approach assuming that QoL improvements with RFCA compared with AADs are (i) maintained for a lifetime (lifetime analysis) or (ii) maintained for a maximum of 5 years only (5year analysis). Second, the base-case analysis uses estimates of the primary outcome (that is, freedom from arrhythmia at 12 months) from the RCT evidence alone. The impact of incorporating additional observational evidence for RFCA from the survey data and the individual case-series is investigated for a subgroup of patients with a baseline risk of stroke equivalent to $\mathrm{CHADS}_{2}=1$. Third, the prognostic value of NSR is examined. The base-case assumes that restoration to NSR results in a relative risk reduction of stroke compared to AF. In an alternative scenario, the risk of stroke is assumed to be 
Table 2 Cost-effectiveness results for the base-case analysis

\begin{tabular}{|c|c|c|c|c|c|c|}
\hline & \multirow[b]{2}{*}{ Strategy } & \multirow{2}{*}{$\begin{array}{l}\text { Mean costs } \\
\text { (f) }\end{array}$} & \multirow[b]{2}{*}{ Mean OALYs } & \multirow[b]{2}{*}{ ICER (f) } & \multicolumn{2}{|c|}{$\begin{array}{l}\text { Probability of being cost- } \\
\text { effective for threshold at: }\end{array}$} \\
\hline & & & & & f20 000 & f30 000 \\
\hline \multicolumn{7}{|c|}{ Lifetime analysis } \\
\hline \multirow[t]{2}{*}{$\mathrm{CHADS}_{2}=0$} & RFCA & 25240 & 12.37 & 7763 & 0.983 & 0.996 \\
\hline & AADs & 14415 & 10.98 & - & & \\
\hline \multirow[t]{2}{*}{$\mathrm{CHADS}_{2}=1$} & RFCA & 26027 & 12.14 & 7780 & 0.981 & 0.996 \\
\hline & AADs & 15367 & 10.77 & - & & \\
\hline \multirow[t]{2}{*}{$\mathrm{CHADS}_{2}=2$} & RFCA & 26987 & 11.87 & 7765 & 0.986 & 0.999 \\
\hline & AADs & 16517 & 10.52 & - & & \\
\hline \multirow[t]{2}{*}{$\mathrm{CHADS}_{2}=3$} & RFCA & 28343 & 11.49 & 7910 & 0.992 & 1.000 \\
\hline & AADs & 18107 & 10.19 & - & & \\
\hline \multicolumn{7}{|l|}{ 5-year analysis } \\
\hline \multirow[t]{2}{*}{$\mathrm{CHADS}_{2}=0$} & RFCA & 25251 & 11.35 & 27745 & 0.091 & 0.577 \\
\hline & AADs & 14429 & 10.96 & - & & \\
\hline \multirow[t]{2}{*}{$\mathrm{CHADS}_{2}=1$} & RFCA & 26016 & 11.18 & 25510 & 0.165 & 0.686 \\
\hline & AADs & 15352 & 10.76 & - & & \\
\hline \multirow[t]{2}{*}{$\mathrm{CHADS}_{2}=2$} & RFCA & 26972 & 10.97 & 23202 & 0.265 & 0.786 \\
\hline & AADs & 16499 & 10.52 & - & & \\
\hline \multirow[t]{2}{*}{$\mathrm{CHADS}_{2}=3$} & RFCA & 28366 & 10.67 & 20831 & 0.418 & 0.881 \\
\hline & AADs & 18133 & 10.18 & - & & \\
\hline
\end{tabular}

$\mathrm{AAD}$, anti-arrhythmic drug; $\mathrm{CHADS}_{2}$, congestive heart failure, hypertension, age 75, diabetes mellitus, and prior stroke; ICER, incremental cost-effectiveness ratio; QALY, quality-adjusted life year; RFCA, radiofrequency catheter ablation.

identical for both treatments and therefore the subsequent costeffectiveness is based entirely on the symptomatic benefit of RFCA compared to AADs. Fourth, the base-case analysis which applies separate utility estimates to patients in the NSR and AF health states according to treatment received is relaxed and no differential impact on $\mathrm{QoL}$ according to treatment received is assumed - that is, separate utility estimates are assigned to NSR and AF (NSR > AF) but assumed to be the same for RFCA and AADs. Fifth, the annual transition probability of reversion back to AF for patients receiving RFCA was increased to $5-15 \%$ per annum. Any additional benefit or reduction in costs assigned to NSR relative to AF is only maintained in the long term if patients continue to remain free of arrhythmia.

\section{RESULTS}

\section{Base-case analysis}

Table 2 presents the base-case results according to the baseline risk of stroke and maintenance time of QoL improvements. For the lifetime analysis, the ICER associated with RFCA compared with AADs is between $£ 7763$ and $£ 7910$ for each additional OALY across the different $\mathrm{CHADS}_{2}$ scores. The probability that RFCA is cost-effective at thresholds of $£ 20000$ and $£ 30000$ per OALY varies from 0.981 to 0.992 and from 0.996 to 1.000 , respectively, across the separate risk groups. Hence there is little uncertainty that RFCA is the optimal treatment if QoL benefits are maintained over a lifetime and the NHS is prepared to pay this amount.

For the 5-year analysis, the ICER associated with RFCA compared with AADs is between $£ 20831$ and £27 745 per OALY gain across the separate groups, with the cost-effectiveness appearing more favourable the higher the risk of stroke. The probability that RFCA is cost-effective at thresholds of $£ 20000$ and $£ 30000$ per OALY is much lower (ranging from 0.091 to 0.418 and from 0.577 to 0.881 , respectively) compared to the lifetime analysis. Hence, the cost-effectiveness of RFCA is less clear if the QoL improvements are maintained for a maximum of 5 years only.

Table 3 Cost-effectiveness of RFCA for alternative scenarios (for $\mathrm{CHADS}_{2}=1$ )

\begin{tabular}{|c|c|c|c|c|c|c|}
\hline & \multicolumn{3}{|c|}{ Lifetime analysis } & \multicolumn{3}{|c|}{ 5-year analysis } \\
\hline & ICER (f) & $\begin{array}{l}\text { Probability CE at } \\
\text { f20 } 000\end{array}$ & $\begin{array}{l}\text { Probability CE at } \\
\text { f30 } 000\end{array}$ & ICER (f) & $\begin{array}{l}\text { Probability CE at } \\
\text { f20 } 000\end{array}$ & $\begin{array}{l}\text { Probability CE at } \\
\text { £30000 }\end{array}$ \\
\hline RCTs and worldwide survey ${ }^{9}$ & 7814 & 0.988 & 1.000 & 25623 & 0.156 & 0.701 \\
\hline RCTs and case-series & 7851 & 0.975 & 0.999 & 25573 & 0.184 & 0.634 \\
\hline \multicolumn{7}{|l|}{ Prognostic impact of NSR } \\
\hline Prognosis for NSR and AF equivalent & 9327 & 0.977 & 0.996 & 37997 & 0.027 & 0.204 \\
\hline No differential impact by treatment & 12840 & 0.682 & 0.963 & 32524 & 0.008 & 0.399 \\
\hline \multicolumn{7}{|c|}{$\begin{array}{l}\text { Annual probability of reversion back to AF after } \\
\text { RFCA }\end{array}$} \\
\hline (a) $5 \%$ & 7999 & 0.982 & 0.999 & 26969 & 0.132 & 0.618 \\
\hline (b) $10 \%$ & 8401 & 0.966 & 0.970 & 29910 & 0.075 & 0.441 \\
\hline (c) $15 \%$ & 8703 & 0.954 & 0.944 & 32035 & 0.060 & 0.374 \\
\hline
\end{tabular}

$\mathrm{AF}$, atrial fibrillation; CE, cost-effectiveness; ICER, incremental cost-effectiveness ratio; NSR, normal sinus rhythm; QALY, quality-adjusted life year; RCT, randomised controlled trials; RFCA, radiofrequency catheter ablation. 


\section{Alternative scenarios}

Table 3 presents the results for alternative scenarios. The inclusion of observational and case-series evidence for RFCA results in only a marginal increase in the ICER for both the lifetime and 5-year analysis. The prognostic impact of NSR results in a greater influence on the cost-effectiveness of RFCA. In the lifetime analysis, the ICER increased from $£ 7780$ (basecase) to $£ 9237$ per additional OALY, while for the 5-year analysis it increased from £25 510 to 337 997. These results would indicate that the cost-effectiveness of RFCA requires either the QoL benefits to be maintained for more than 5 years and/or the assumption that NSR has prognostic value in preventing the risk of stroke. The alternative assumption of no differential impact on OoL according to treatment received, except through entry into the NSR and AF health states, had an impact on the cost-effectiveness of RFCA. The ICER of RFCA in the lifetime analysis increased to $£ 12840$ per additional OALY, while the ICER for the 5-year analysis increased to £32 524 per additional OALY. Consequently, the results indicate that the cost-effectiveness of RFCA is sensitive to the assumption of a differential utility estimate for NSR and AF according to treatment received. If it is believed that RFCA confers additional QoL benefits to patients following a successful treatment compared to patients receiving $\mathrm{AADs}$ and/or that the $\mathrm{OoL}$ benefits are maintained for more than 5 years, then the costeffectiveness of RFCA is less uncertain. Increasing the long-term risk of recurrent AF following RFCA results in a marginal increase in the ICER in the lifetime analysis. In the 5-year analysis, when the annual probability is increased to $15 \%$, the ICER is above the range considered to be cost-effective.

\section{DISCUSSION}

Only one previous study has examined the cost-effectiveness of RFCA for AF. ${ }^{4}$ This study is based on a US population and as such it has a number of limitations for decision-making in the UK NHS. For example, in the United Kingdom the pattern of care and number of cardiologists undertaking RFCA differs from the United States. In addition, the majority of patients referred for RFCA therapy in the United Kingdom have failed previous $\mathrm{AAD}$ therapy and, therefore, unlike the United States, RFCA is unlikely to be given as a possible first-line therapy. The present study provides a comprehensive assessment of the costeffectiveness of RFCA for patients refractory to AADs in the UK NHS. Unlike the one previous study, ${ }^{4}$ this analysis attempts to capture the additional symptomatic benefits in addition to any additional prognostic benefit.

The results of the analysis presented here demonstrate that the long-term maintenance of QoL benefits of RFCA is central to the cost-effectiveness estimates. If the benefits are maintained over the lifetime of the patient, RFCA is likely to be highly cost-effective based on the cost-effectiveness thresholds used by the National Institute for Health and Clinical Excellence (£20 000 to $£ 30000$ per additional OALY). ${ }^{25}$ This conclusion also holds across a range of different baseline risks of stroke. The finding contrasts with the previous study, ${ }^{4}$ where RFCA was found to be cost-effective only in those at high risk of stroke and in certain patients groups with moderate stroke risk. This difference highlights the importance of symptomatic QoL benefits. If the assumption of lifetime benefits is considered unrealistic then the question of how long the benefits are likely to be maintained becomes a key consideration. The results of the 5-year analysis suggest that the cost-effectiveness of RFCA is not clearcut with an ICER of $£ 25510$ falling just below the upper bound of conventional thresholds. Any shorter duration of QoL benefits would result in an ICER above acceptable thresholds (for example, 4-year duration results in an ICER = $£ 30$ 102; 3-year duration, ICER = $\$ 37$ 385; 2-year duration, ICER $=£ 49$ 355).

The results from the different scenarios suggest that the overall cost-effectiveness of RFCA for the shorter duration of benefits is likely to be determined by a number of factors. These include: (i) whether there are additional prognostic benefits associated with NSR (that is, via a reduction in the long-term risk of stroke); and (ii) the magnitude of the QoL difference between RFCA and AADs. The importance of these factors will decline the longer any QoL advantage associated with RFCA is maintained beyond 5 years.

While the cost-effectiveness model attempted to quantify the potential OoL gains that could be achieved using RFCA through symptomatic improvements and reduction in the longer-term risk associated with major clinical events, a number of limitations to the analysis should be noted. First, the QoL estimates applied in the model remain highly uncertain. To date no single study has attempted to quantify the impact of RFCA using a generic, utility measure such as the EQ-5D. This represents a major limitation when trying to establish the costeffectiveness of an intervention in the NHS. In the absence of these data, alternative approaches were used to attempt to map between the QoL measures that have been used (SF-36) and a utility based measure (EO-5D). The process of mapping between these different instruments itself introduces a source of uncertainty. However, a number of separate scenarios were considered in the model and these demonstrated that the overall results remained fairly robust to the different estimates, suggesting that the duration of any benefits is likely to be the key determinant of cost-effectiveness. Second, the evidence of the longer-term benefits of RFCA (that is, for periods potentially beyond 5 years) is lacking and hence extrapolating the potential benefits reported over shorter time horizons becomes increasingly uncertain. The model results clearly demonstrate that the cost-effectiveness estimates are extremely sensitive to the duration over which these benefits are likely to be maintained. Third, in the absence of direct evidence from RCTs, the AFFIRM study was used to quantify the potential differential stroke risk for NSR/AF but this study did not involve RFCA. Fourth, the decision model only considers the cost-effectiveness of RFCA in patients with predominantly paroxysmal AF.

In conclusion, the analysis suggests that RFCA is potentially cost-effective for the treatment of paroxysmal AF in patients predominantly refractory to AAD therapy if the QoL benefits are maintained for more than 5 years. These findings remain subject to important uncertainties regarding both the magnitude of QoL benefits and the prognostic value of restoring NSR achieved with RFCA.

Funding: This project was funded by the National Institute for Health Research (NIHR) Health Technology Assessment Programme. The views expressed are those of the authors who are also responsible for any errors.

Competing interests: None.

\section{REFERENCES}

1. Steinberg JS. Atrial fibrillation: an emerging epidemic? Heart 2004;90:239-40.

2. Stewart S, Murphy N, Walker A, et al. Cost of an emerging epidemic: an economic analysis of atrial fibrillation in the UK. Heart 2004;90:286-92.

3. National Institute for Health and Clinical Excellence. Percutaneous radiofrequency ablation for atrial fibrillation. London: NICE, 2006:1-2.

4. Chan PS, Vijan S, Morady F, et al. Cost-effectiveness of radiofrequency catheter ablation for atrial fibrillation. J Am Coll Cardiol 2006;47:2513-20. 
5. National Institute for Clinical Excellence. Guidance to the methods of technology appraisal. London: NICE, 2004

6. Krittayaphong R, Raungrattanaamporn 0 , Bhuripanyo $\mathrm{K}$, et al. A randomized clinical trial of the efficacy of radiofrequency catheter ablation and amiodarone in the treatment of symptomatic atrial fibrillation. J Med Assoc Thai 2003;86(suppl 1):S816.

7. Pappone C, Augello G, Sala S, et al. A randomised trial of circumferential pulmonary vein ablation versus antarrhythmic drug therapy in paroxysmal atrial fibrillation. $J$ Am Coll Cardiol 2006;48(11).

8. Wazni OM, Marrouche NF, Martin DO, et al. Radiofrequency ablation vs antiarrhythmic drugs as first-line treatment of symptomatic atrial fibrillation: a randomized trial. JAMA 2005;293:2634-40.

9. Cappato R, Calkins H, Chen SA, et al. A worldwide survey on the methods, efficacy, and safety of catheter ablation of atrial fibrillation. Circulation 2004;110:2524.

10. Pappone C, Rosanio S, Augello G, et al. Mortality, morbidity, and quality of life after circumferential pulmonary vein ablation for atrial fibrillation: outcomes from a controlled nonrandomized long-term study. J Am Coll Cardiol 2003;42:185-97.

11. Roy D, Talajic M, Dorian P, et al. Amiodarone to prevent recurrence of atrial fibrillation. Canadian Trial of Atrial Fibrillation Investigators. N Engl J Med 2000:342:913-20.

12. Gage $\mathbf{B F}$, Waterman $A D$, Shannon $W$, et al. Validation of clinical classification schemes for predicting stroke: results from the National Registry of Atrial Fibrillation. JAMA 2001;285:2864-70

13. Lip GY, Edwards SJ. Stroke prevention with aspirin, warfarin and ximelagatran in patients with non-valvular atrial fibrillation: a systematic review and meta-analysis. Thromb Res 2006:118:321-33.

14. Sherman DG, Kim SG, Boop BS, et al. Occurrence and characteristics of stroke events in the Atrial Fibrillation Follow-up Investigation of Sinus Rhythm Management (AFFIRM) study. Arch Intern Med 2005;165:1185-91.

15. Nieuwlaat R, Capucci A, Lip GYH, et al. Antithrombotic treatment in real-life atrial fibrillation patients: A report from the Euro Heart Survey on Atrial Fibrillation. Eur Heart J 2006;27:3018-26.

16. Dennis MS, Burn JP, Sandercock PA, et al. Long-term survival after first-ever stroke: the Oxfordshire Community Stroke Project Stroke 1993;24:796-800.

17. Government Actuary's Department. Life Tables 2007.

18. Department of Health. Reference costs. Leeds: DH, 2005.
19. Jones L, Griffin S, Palmer S, et al. Clinical effectiveness and cost-effectiveness of clopidogrel and modified-release dipyridamole in the secondary prevention of occlusive vascular events: a systematic review and economic evaluation. Health Technol Assess 2004;3:1-196.

20. Kind P, Hardman G, Macran S. UK population norms for EQ-5D. Centre for Health Economics discussion paper 172. York: Centre for Health Economics, University of York, 1999.

21. Sullivan $\mathbf{P}$, Ghushchyan V. Preference-based EQ-5D index scores for chronic conditions in the US. Med Decis Making 2006:26:410-420.

22. Owens DK, Sanders GD, Harris RA, et al. Cost-effectiveness of implantable cardioverter defibrillators relative to amiodarone for prevention of sudden cardiac death. Ann Intern Med 1997;126:1-12.

23. Johannesson M, Weinstein S. On the decision rules of cost-effectiveness analysis. $J$ Health Econ 1993:12:459-67.

24. Bourke JP, Dunuwille A, O'Donnell D, et al. Pulmonary vein ablation for idiopathic atrial fibrillation: six month outcome of first procedure in 100 consecutive patients. Heart 2005;91:51-7.

25. Devlin N, Parkin D. Does NICE have a cost effectiveness threshold and what other factors influence its decisions? A binary choice analysis Health Econ 2004;13:43752.

26. National Institute for Health and Clinical Excellence. Atrial fibrillation: the management of atrial fibrillation. NICE clinical guideline 36. London: NICE, 2006.

27. Berkowitsch A, Neumann T, Kurzidim K, et al. Comparison of generic health survey SF-36 and arrhythmia related symptom severity check list in relation to post-therapy AF recurrence. Europace 2003;5:351-5.

28. Rienstra M, Van Gelder IC, Hagens VE, et al. Mending the rhythm does not improve prognosis in patients with persistent atrial fibrillation: a subanalysis of the RACE study. Eur Heart J 2006;27:357-64.

29. British Medical Association, Royal Pharmaceutical Society of Great Britain British National Formulary: No 53, March 2007. London: BMA, Royal Pharmaceutical Society of Great Britain, 2007.

30. Buxton M, Caine N, Chase D, et al. A review of the evidence on the effects and costs of implantable cardioverter defibrillator therapy in different patient groups, and the modelling of cost-effectiveness and cost-utility for these groups in a UK context. Health Technol Assess 2006;10:1-180. 